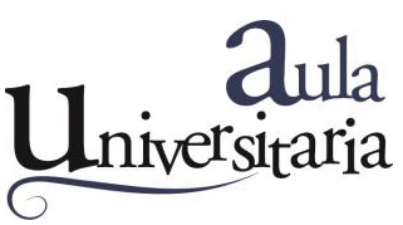

\title{
El programa de tutoría como facilitador del ingreso universitario
}

\author{
FASCENDINI, Paola Y.; GUAITA, María L.;1 OJEDA, Norberto²
}

\author{
Filiaciones institucionales \\ ${ }^{1}$ Servicio Orientación Educativa. Facultad de Ciencias Veterinarias. Universidad Nacional del Litoral. \\ ${ }^{2}$ Médico Veterinario. Facultad de Ciencias Veterinarias. Universidad Nacional del Litoral.
}

\section{Correspondencia}

Facultad de Ciencias Veterinarias. Universidad Nacional del Litoral. P. Kreder 2805 (3080) Esperanza, Santa Fe. Argentina. Tel. 54-3496-420639

pfascendini@fcv.unl.edu.ar

\section{Resumen}

El presente trabajo busca visibilizar el impacto del Programa de Tutorías que se lleva a cabo en el ingreso universitario de la carrera de Medicina Veterinaria en la Facultad de Ciencias Veterinarias, en un trabajo compartido entre la cátedra de Introducción a la Veterinaria y el Servicio de Orientación Educativa (SOE). El objetivo del Programa es acompañar a los estudiantes para favorecer no solo su ingreso al mundo superior sino también brindar espacios para adquirir las habilidades cognitivas necesarias, en la conquista del logro de sus aprendizajes. Se aplicó una encuesta a 126 estudiantes de la cohorte 2018, ingresantes a la carrera, y participantes del Programa de Tutorías. Teniendo en cuenta los resultados, el Programa de Tutorías es recomendado por el $93 \%$ de los tutorados debido a su eficacia en los procesos de afiliación universitaria. Es llevado a cabo por estudiantes avanzados, quienes, tras un proceso de selección y capacitación, realizan la tarea orientativa. Además, los tutores reconocen los beneficios del acompañamiento a otros en su permanencia institucional y refieren un antes y después en la participación realizada, teniendo en cuenta los cambios significativos que observan en sí mismos.

\section{Palabras clave \\ ingreso | Programa de Tutorías | prevención | estudiantes | universidad}




\section{Summary}

The present work search to make visible the impact of the Mentoring Program that is carried out in the university entrance of the Veterinary Medicine career at the Faculty of Veterinary Sciences, in a shared work between the Chair of Introduction to Veterinary Medicine and the Guidance Service Educational (SOE). The objective of the Program is to accompany students to favor not only their entrance into the higher world, but also to provide spaces to acquire the necessary cognitive skills, in the conquest of their learning achievement. A survey was applied to 126 students from the 2018 cohort, entered the race, and students from the Mentoring Program. Taking into account the results, the tutoring program is recommended by $93 \%$ of the tutors due to its effectiveness in the university affiliation processes. The program is carried out by advanced students, who, after a selection and training process, carry out the orientation task. In addition, tutors recognize the benefits of accompanying others in their institutional permanence and affected before and after in the participation carried out, taking into account the significant changes they see in themselves.

\section{Keywords}

entry / Tutorial Program / prevention / students / university

\section{Introducción}

La acción tutorial funda el papel primordial en gestionar procesos de inclusión educativa no solo en términos de acceso, permanencia y egreso, sino también de promoción del reconocimiento del otro como sujeto de derechos, cuyos valores e intereses tendrían que ser incorporados. Este proceso no puede ser pensado al margen de lo sujetos que aprenden, de sus capacidades, posibilidades, aspiraciones e intenciones, como tampoco puede permanecer fuera de las prácticas y de los contextos institucionales, sociales, culturales y políticos en los que los protagonistas actúan y se educan. La educación siempre tiene que ver con las personalidades de los alumnos y el ambiente social que se conforma (Baeza, 2001). Por ello, resulta primordial atender a estas necesidades mediante dispositivos institucionales que permitan acompañar el proceso del ingreso universitario, siendo el tutor un actor central de la transformación institucional (Badillo, 2007). El Programa de Tutorías de la Facultad de Ciencias Veterinarias (FCV) de la Universidad Nacional del Litoral (UNL) surgió en 2010 como una respuesta a las necesidades de los estudiantes que requieren ser acompañados en el proceso de formación. Este Programa, de tutores pares, está destinado a ingresantes de la carrera de Medicina Veterinaria en el primer semestre de cada año, en conjunto con los cursos de articulación disciplinargeneral y las materias del primer año de la carrera. Se orienta a acompañar el ingreso estudiantil y guiar a los nuevos estudiantes en el proceso de adaptación universitaria con el fin de que se apropien lo más precozmente de las habilidades y competencias que deben poseer para alcanzar una eficaz adaptación al ámbito estudiantil. Las tutorías son desarrolladas por estudiantes avanzados de la 
carrera a los cuales se les designa un grupo de 15 estudiantes ingresantes con la coordinación de la cátedra de Introducción a la Veterinaria y SOE (Servicio de Orientación Educativa) de la FCV. El programa se asienta en una metodología vivencial, práctica, mediante la acción interrelacional de un coordinador de tutores que guía su intervención, enlazado por apoyos brindados por el SOE, que cumple la misión de colaborar y personalizar la escucha de las problemáticas individuales a la vez que vierte sugerencias y orientaciones específicas a las necesidades que aquejan a cada estudiante, solidificando la escucha y propiciando el acontecer personalizado (Fascendini, 2016). Los objetivos generales que dan sustento al Programa priorizan la necesidad de incorporación de la tutoría como una estrategia permanente que colabora a disminuir el impacto del fenómeno de deserción y el desgranamiento del ingreso. Para poder evaluar su funcionalidad se ha diseñado una encuesta valorativa junto a entrevistas individuales que permiten recoger no solo datos cuantitativos sino también cualitativos en el ejercicio del rol, tanto de los tutores como de los tutorados.

\section{Objetivos generales del Programa de Tutorías}

Este programa tiene como objetivos primordiales los siguientes:

- Incorporar el sistema de tutoría universitaria como una estrategia permanente que ayude a disminuir el impacto del fenómeno de deserción y desgranamiento de estudiantes.

- Revalorizar el vínculo personal y el aprendizaje en grupo como instancia válida para mejorar el rendimiento en el nivel universitario.

- Establecer a la tutoría como referente relacional facilitadora de la integración académico-social de los ingresantes.

\section{Objetivos específicos}

- Mejorar el desempeño de los estudiantes a través de acciones que apunten a solidificar herramientas metacognitivas.

- Acompañar a los ingresantes mediante la detección de aquellos que se encuentran en una situación límite o crítica. 


\section{Metodología}

La metodología de abordaje que el Programa de Tutorías sustenta se circunscribe a las acciones de un coordinador de tutores que guía la intervención enlazado por apoyos brindados por el SOE. Los tutorados consolidan grupos tutoriales de trabajo que son asignados por un coordinador, habiendo aplicado previamente entrevistas de selección y una posterior capacitación, sabiendo que el tutor debe tener la capacidad efectiva para llevar a cabo exitosamente una actividad laboral plenamente identificada vinculada al liderazgo, creatividad, habilidad verbal, tipo de motivación, relaciones interpersonales y comunicatividad, el que tendrá a su cargo a 15 tutorados ingresantes a la carrera de Medicina Veterinaria. En el marco del Programa de Tutorías y en la instancia de selección de tutores se prevé la aplicación de variadas técnicas que permitan dar cuenta de estos elementos y que sirvan a la hora de la selección, siendo su objetivo principal evaluar a los estudiantes más competentes para el ejercicio del rol tutorial (Gismero González, 2010). La convocatoria estuvo dirigida a estudiantes avanzados en la carrera que debían reunir ciertos requisitos formales, tales como tener el $75 \%$ de las asignaturas del primer año aprobadas y regularizadas, el $80 \%$ del Ciclo Básico de la carrera de Medicina Veterinaria, como mínimo, y contar con un desempeño académico satisfactorio en el último año, además de otros requerimientos vinculados a la presentación de antecedentes y curriculum, sumados a una valoración cualitativa de aptitudes para el ejercicio de este rol tutorial. La selección se realizó en dos etapas; en la primera se valoró el desempeño académico, además del curriculum de los aspirantes, en la segunda etapa se efectuó una entrevista focalizada semiestructurada donde se aplicó una batería de pruebas proyectivas y de habilidades sociales, buscando obtener a través de ellas los perfiles psicológicos más acordes para el desempeño del rol. Para medir las habilidades sociales se instrumentó este año un cuestionario denominado EHS (Escala de Habilidades Sociales) compuesto por 33 ítems, los que exploran la conducta habitual del sujeto en situaciones concretas y valora hasta qué punto las habilidades sociales modulan estas actitudes (Gismero González, 2010). El análisis de las variables revela la presencia de seis factores relacionados con autoexpresión en situaciones sociales, los que reflejan la capacidad para expresarse en forma espontánea y sin ansiedad, el factor defensa de los derechos como consumidor que evidencia la expresión de conductas asertivas frente a desconocidos, la expresión de disconformidad en la que subyace la idea de evitar conflictos o confrontar con otros, el decir no y cortar interacciones, reflejando la habilidad para sostener una conversación acorde, hacer peticiones y el iniciar interacciones positivas con el sexo opuesto. Además, permite elaborar un perfil de estas habilidades mediante la valoración de un índice global del nivel de las mismas (Gismero, 2000). Este índice se divide en cinco categorías según las puntuaciones obtenidas: Alto, Medio Alto, Medio, Medio Bajo y Bajo (Albajari, 2005). Luego se tabulan los datos y se elabora una ficha por orden de mérito 
para establecer una especie de escala en el ordenamiento jerárquico que no deja afuera a nadie en las instancias de capacitación que a continuación se brindan, con el propósito de que en un futuro los que han quedado fuera de mérito vuelven a participar. Los tutores realizan encuentros semanales donde desarrollan actividades previamente programadas en reuniones de coordinación y mediatizadas por material planificado teniendo en cuenta las necesidades y demandas requeridas. De modo paralelo a los encuentros tutoriales, los tutores designados al Programa se reúnen quincenalmente con el coordinador para fundar las actividades, plantear situaciones pertinentes al bienestar estudiantil, sugerencias, y replantear las actividades desarrolladas, hacer análisis de las variables grupales, detección de roles puestos en la tarea concreta, reorientación de estrategias, para conseguir una constante adaptación a las necesidades de los ingresantes universitarios y siendo sus requerimientos particulares a cada grupo en cuestión. Además, se ofrece a los tutorados desde el SOE, junto con el coordinador de las tutorías y docentes que conforman la cátedra de Introducción a la Veterinaria, espacios alternativos y formativos para los tutores, quienes acercan en forma quincenal las necesidades particulares que son vertidas como un modo de reflexionar conjuntamente los procesos que van emergiendo en todo trabajo grupal, sabiendo que el mismo es una construcción que requiere de intervenciones frecuentes que hacen al seguimiento del tutor.

\section{Los contenidos trabajados}

Los encuentros semanales de tutorías se vinculan con una metodología de abordaje de tipo taller, donde la selección de algunas temáticas esenciales detectadas en los ingresos de los estudiantes y observadas en verbalizaciones constantes y consultas permanentes se vinculan con la cuestión de la organización temporal del estudio, en los aspectos organizativos y administrativos de la institución, en las estrategias de estudio, en el interés y motivación, en la contención afectiva, producción de textos académicos en el contexto de diversas disciplinas, entre otros. Además, se complementa dicho programa con la labor de interacciones grupales y personales acerca de demandas que aparecen en los aconteceres individuales para que, por intermedio del SOE, puedan ser canalizados y acompañados en forma sistemática, sabiendo que dicho espacio, como verbalizan los estudiantes, es «el lugar donde sentimos que no somos un número para la facultad».

\section{Material y frecuencia}

Un cuadernillo de trabajo articulado con diversas temáticas que resulta didáctico en su presentación visual además de ser interactivo por la autoaplicabilidad y la facilidad para la administración. Los encuentros semanales suelen ser de 90 
minutos, donde la actividad se centraliza en generar un espacio participativo que favorezca el debate, la escucha, la confianza y la posibilidad de verter y canalizar las preocupaciones que emerjan ante este nuevo espacio formativo. E cuadernillo se encuentra subdividido en ejes relacionados con los procesos de adaptación, la organización personal y de estudio, la universidad y sus características, los canales de aprendizaje y los sistemas evaluativos, y ofrece actividades dinámicas y autorreflexivas que permitan fortalecer el conocimiento propio y del entorno formativo en el que se inserta.

\section{Resultados}

La instancia evaluativa del Programa de Tutorías llevada a cabo persigue como objetivo primordial el reconocimiento de logros y obstáculos en la puesta en marcha de este espacio alternativo a los de enseñanza formal, así como conocer la vivencia que han tenido tanto tutores como tutorados en el ejercicio del rol. Para ello se ha diseñado una encuesta valorativa que permite recoger datos en función de la evaluación del ejercicio tutorial y analizar también las voces y vivencias en las experiencias estudiantiles mediatizadas por entrevistas semiestructuradas para dar cuenta del significado otorgado a este dispositivo institucional.

El diseño de la encuesta se organizó en el formato de documento Drive para facilitar su aplicación y fue administrado a 126 estudiantes ingresantes a la carrera de Medicina Veterinaria, quienes participaron de los encuentros tutoriales en el primer semestre 2018. En función de la estructura del recurso diseñado, la misma consta de una serie de interrogantes dirigidos a la funcionalidad del Programa de Tutorías, a la necesidad de brindar continuidad al mismo, a la aplicabilidad de las temáticas enseñadas, a la experiencia realizada. La metodología de abordaje en relación con los tutorados fue llevada a cabo por los tutores, quienes facilitan el acceso a un link para que los ingresantes puedan completarlo con sus opiniones y vivencias. También como un modo de acompañar a los tutores, el SOE llevó a cabo entrevistas personales de seguimiento, con el propósito de acercar un análisis introspectivo y personal.

Teniendo en cuenta las respuestas brindadas, en la dimensión organización universitaria y necesidad de darle continuidad al programa, un 92,9 \% (117/126) de los tutorados respondió favorablemente. Además, resaltaron que este Programa fue un apoyo, acompañamiento constante a los procesos del ingreso y un espacio para aprender estrategias de estudio, constituyéndose en un referente asertivo para el cambio que se avecinaba.

En cuanto al ítem referente a las actitudes evidenciadas en el tutor, el 79,4\% (100/126) de los tutores se manifestó dispuesto; el 73,8 \% (93/126), responsable; el 70,6 \% (89/126), abierto las necesidades; el 68,3 \% (86/126), comprometido, y destacaron valores primordiales en el trato humano y cercano que se propician en el intercambio con el otro. Asimismo, resaltaron la amabilidad, la clarificación 
de las dudas, confiabilidad, empatía, puesta al servicio de su función, benefactoras colaborativas en la orientación educativa como vectores preponderantes, facilitadores de los procesos de cambio y adaptación.

Con respecto a las temáticas desarrolladas en los encuentros tutoriales y el grado de conformidad de los tutorados, el 44,4 \% (56/126) expresó que estuvo muy conforme; y el 49,2\% (62/126), conforme. En el ítem de temáticas de los encuentros, refirieron aplicabilidad de las estrategias de estudio y organizativas enseñadas en el $54 \%$ (68/126) frecuentemente, lo cual evidencia utilidad de lo comunicado y referencialidad positiva para su posterior uso.

Haciendo alusión a una valoración un tanto más cualitativa, y contemplando la pregunta de cómo definirían a la tutoría luego de haber experimentado el Programa, los ingresantes la conceptualizaron como una experiencia maravillosa en la que se puede hacer amigos, conocer compañeros, expresarse, perder el miedo a lo nuevo, y que constituye una guía primordial para el ingreso, conforme al valor que tiene contar con alguien incondicional, siempre presente para cualquier duda o temor, una forma de aprender a trabajar en grupo, interesante, un consejero, una persona que siempre alienta a seguir, entre otras cosas que mencionaron.

\section{La voz de los tutores}

Otra lente sobre la cual se ha mirado a la tutoría son las palabras de los mismos tutores. En la puesta en marcha de este dispositivo, se promovieron espacios personales de análisis y valoración de la propia experiencia como un modo introspectivo de facilitar el enriquecimiento personal. A través de entrevistas individualizadas se impulsó el diálogo ameno para analizar las emociones y experiencia construida. En sus voces, los tutores refieren que hay un antes y un después de las tutorías en su experiencia estudiantil, pues esta incursión les ha posibilitado un incremento de las propias habilidades comunicacionales, ha mejorado la confianza en sí mismos, potenciado su autoestima y favorecido además el aprendizaje con otros; sabiendo que en el ejercicio de esta función han consolidado su crecimiento personal y construido un rol como estudiantes al servicio de otros, como actores sociales activos en el ámbito universitario. En su gran mayoría, redoblan la apuesta y desean repetir la experiencia como un modo de ir ensayando roles ocupacionales, pero fundamentalmente para forjar mejores habilidades en la conformación de sujetos íntegros.

\section{Conclusión}

Pensar en la imagen y figura del tutor es realizar una analogía vinculante con las ciencias de la naturaleza donde, a una planta que recién inicia su proceso de crecimiento, se le ofrece un soporte que oficia de sostén, que hace posible su 
desarrollo y adaptación al medio a través del mejoramiento de las condiciones. Este tutor se encuentra al lado, ni adelante ni atrás, no tiende a realizar los esfuerzos de la planta al crecer, ni soluciona las inclemencias temporales o la aridez del terreno, simplemente está allí al lado, acompañando dicho proceso, y cuando la planta se arraigue a suelo firme, se pensará en la alternativa de sacarla para continuar con su desarrollo (Fascendini, 2016). Es así como el tutor no suple los esfuerzos del estudiante, sino que se convierte en referente que brinda luz y guía los procesos que el mismo tiene que realizar. Es crucial, en el nivel superior, pensar en la incorporación y sostenimiento de dispositivos institucionales vinculados al ejercicio tutorial que se establezcan como estrategias de apoyo preventivas para los estudiantes, con el objetivo de colaborar y encauzar los obstáculos que pudieran atravesar en el ingreso universitario, ofreciendo herramientas personales y de estudio para hacer frente a este cambio inminente. Resulta entonces que el Programa de Tutorías favorece el proceso de afiliación de los estudiantes tutorados en el ingreso y motiva a los estudiantes tutores, que en el ejercicio de su rol fortalecen su permanencia en el sistema educativo. Se rescata como necesaria e indispensable la labor tutorial, no solo por la riqueza que trae aparejada la puesta en marcha del rol, sino también por los intercambios interpersonales que ponen a prueba aptitudes, habilidades, rasgos en la personalidad que permiten madurar psicoafectivamente, pulir asperezas caracterológicas y propiciar espacios de reflexión internos valiosos para la formación humana. Además, en los tutorados se propicia una mayor adaptabilidad al contexto universitario y una vivencia de humanidad incuestionable. La puesta en marcha de esta estrategia institucional tiende a favorecer la adquisición de herramientas fundamentales para el proceso de estudio y aprendizaje y mejorar las habilidades personales y la puesta en práctica de las mismas. La tutoría así se convierte en una estrategia fundamental en la que se permite abordar la construcción del propio proyecto personal de vida (País, 2014) a través de experiencias vitales en general y laborales en particular. En términos vivenciales, el tutor es como un amigo en la ruta de la vida que posibilita otorgar el escudo de la perseverancia, la bandera del optimismo, el estandarte del esfuerzo junto al lema del «yo puedo».

\section{Agradecimientos}

A todos los tutores participantes. A los tutorados. Al equipo de cátedra. 


\section{Referencias bibliográficas}

- Albajari, V. y Names, S. (2005). La evaluación psicológica en selección de personal. Paidós.

- Badillo Guzmán, J. (2007). La tutoría como estrategia viable de mejoramiento de la calidad de la educación superior. Reflexiones en torno al curso CPU-e. Revista de Investigación Educativa, (5), 1-22.

- Baeza, J. (2001). El oficio de ser alumno en jóvenes de liceo de sector popular. Santiago: Universidad Católica Cardenal Raúl Silva Henríquez. Revista Electrónica Diálogos Educativos, 2(3), 88.

- Fascendini, P.Y. (2016). Estrategias metacognitivas: el ingreso a la universidad. ¿Cómo aprende el que aprende? (37-39). Editorial Académica Española.

- Gismero González, E. (2000). Escala de habilidades sociales. Tea.

- Pais, H. (2014). Tutoría con adolescentes en la nueva escuela secundaria. Universidad Católica de Santa Fe. 